\title{
The Effects of Kinesio-taping Applied to Delayed Onset Muscle Soreness on Changes in Pain
}

\author{
Sea-Hyun Bae ${ }^{1}$, Young-Shin Lee ${ }^{3}$, Gi-Do Kim ${ }^{2}$ and Kyung-Yoon $\mathrm{Kim}^{3}$ \\ ${ }^{1}$ Department of Physical Therapy Cheongam University, Suncheon, Korea, \\ ${ }^{2}$ Department of Physical Therapy International University, Jinju, Korea, \\ ${ }^{3}$ Department of Physical Therapy Dongshin University, Naju, Korea \\ 'qbseadp@daum.net, ${ }^{2}$ pray1980@daum.net, \\ ${ }^{3}$ lys3231@naver.com, ${ }^{3}$ redbead7@daum.net
}

\begin{abstract}
This study aimed to examine changes in pain over time through quantitative sensory testing (QST) when delayed onset muscle soreness (DOMS) was artificially induced on the non-dominant biceps brachii. Thirty-three healthy males to whom DOMS was induced through eccentric exercise among those who participated in this study were randomly assigned to group 1 ( sham taping, $n=17$ ) and group II (kinesio taping, $n=16$ ).

Kinesio taping was applied in the same driving direction as that of muscle fibers. QST was measured at four time points (prior to DOMS induction, 24 hours after DOMS induction, 48 hours after DOMS induction, and 72 hours after DOMS induction) and there were significant differences in cold pain threshold (CPT), warm pain threshold (WPT), and cold sensation threshold (CST) 24 hours after DOMS induction $(p<0.05)$ and 48 hours after DOMS induction $(p<0.05)$ compared to prior to DOMS induction. The threshold values of group II to whom kinesio taping was applied did not significantly differ between prior to DOMS induction and 72 hours after DOMS induction ( $p>0.05)$. There were statistically significant differences in CST and WST between the two groups 72 hours or after the intervention $(p>0.05)$.

Visual analogue scale (VAS) that represents subjective pain degree according to time of measurement was measured; VAS started to increase 24 hours after DOMS induction and became largest 48 hours after DOMS induction. Pain statistically significantly decreased 72 hours after DOMS induction relative to 24 hours after DOMS induction ( $p<0.05)$.

The effects of Kinesio taping on decrease in DOMS according to measured time were examined through QST and its usefulness was verified.
\end{abstract}

Keywords: Delayed onset muscle soreness (DOMS), Biceps brachii, Kinesio taping, Quantitative Sensory Testing (QST)

\section{Introduction}

Delayed onset muscle soreness (DOMS) is muscle pain that occurs when unfamiliar movements such as sudden and violent physical activities of high intensity are performed [1]. In particular, DOMS is likely to occur during eccentric contraction of the muscles when going down the stairs and unloading heavy objects [2]. DOMS takes place within 24 hours after exercise, becomes maximal between 48 and 72 hours, and disappears between five and seven days after its initiation [3]. In addition, its symptoms such as reduction in range of motion, dull pain such as swelling, decrease in muscle strength, and muscle spasticity affect activities of daily living [4]. 
Pathogenesis of DOMS is not clear. Metabolic waste accumulation theory [5] explains that lactic acid accumulates after exercise, triggering pain. According to muscle spasm theory [6], pain and muscle spasm are caused by ischemia or metabolic waste products during exercise. Microtrauma theory [7] views that DOMS is triggered by inflammation and tissue degeneration after micromechanical damage of muscle fibers or connective tissues due to movements. DOMS is similar to acute inflammatory response of the muscles through proliferation of macrophages or increase in lysosome activity [8].

In order to prevent and treat DOMS, massage, stretching, warm-up exercise, cool-down exercise, electrical stimulation, ultrasound, cold therapy, and drug therapy have been performed [1,9]. However, only massage was effective in slightly reducing relevant symptoms [10]. In addition, due to its subjective traits, expressing DOMS into numerical values through tools and methods that measure muscle pain is difficult [11]. Therefore, methods to treat and prevent DOMS and quantitative tools to measure their effects are needed.

Physical therapists have employed taping in order to alleviate pain or prevent injuries and their recurrence in the rehabilitation or sports area [12]. Kenzo Kase complemented adhesive tapes and developed them into elastic tapes; They are attached to legions and muscles around them as a treatment method [13].

Elastic Kinesio taping is widely used not only for patients but also for the purpose of preventing damages. Generally, it improves motor functions by inhibiting hypertonic muscles and stimulating hypotonic muscles, thereby enhancing proprioceptive senses, optimizing joint alignment, decreasing load on hypersensitive nerve tissues, and reducing pain [14] as well as enhancing muscle functions such as muscle strength, endurance, and power [15].

Pain is assessed using scale methods, such as simple descriptive scale, Visual analog scale (VAS), verbal rating scale (VRS), and subjective methods such as McGill pain questionnaire and dallas pain questionnaire; Research to objectify such subjective methods is being conducted [16]. VAS is commonly used as the most appropriate tool to evaluate pain intensity depending on patients' subjective judgement [17]. Nonetheless, it is a subjective measurement method and has ambiguous aspects in an evaluator's analysing the results [18].

For pain evaluation, reliable ground for a patient's conditions should be provided and smooth communications between the tester and the patient should be made. The importance of Quantitative Sensory Testing (QST) is being emphasized for the obtaining of objective and specific assessment data and reliable evaluation.

QST is to assess functions of large and small nerve fibers and is known as a useful method to evaluate changes in diverse somatic senses [19]. QST is relatively simple and non-invasive, and the examinee may endure it with ease. Temperature threshold test, an essential item of QST, examines cold sensation threshold (CST), warm sensation threshold (WST), cold pain threshold (CPT), and warm pain threshold (WPT), thereby evaluating the degree of damage in A-delta and C fibers; Reliability of the test has been proved [20, 21].

Therefore, this study intended to look at changes in pain by quantifying the effects of Kiensio-taping application according to measured time point through QST as a method to objectify DOMS whose assessment had depended on subjective evaluation.

\section{Subjects and Methods}

\subsection{Selection of the Subjects}

This study randomly assigned 33 healthy male adults in their 20s who experienced DOMS after eccentric contraction exercise among 40 participants to group I and group II using double blind method. The criteria for inclusion were: those who had no restriction in joint 
movement; those who had not conducted regular exercise for the past three months; those whose dominant arm was the right arm; those who had no open wound and inflammatory disease in the measured area; and those who did not take medication affecting the experiment. Data collection was initiated after approval from Dongshin University Institutional Review Board was obtained. All the subjects of this study voluntarily consented to participate in this study. Their characteristics are shown in Table 1.

Table 1. Characteristics of Study Participants

\begin{tabular}{ccc}
\hline Parameters & Group I & Group $\Pi$ \\
\hline Age (years) & $24.71 \pm 3.25$ & $25.10 \pm 2.60$ \\
Height $(\mathrm{cm})$ & $176.19 \pm 10.70$ & $174.26 \pm 9.40$ \\
Weight $(\mathrm{kg})$ & $71.25 \pm 9.22$ & $68.24 \pm 4.30$ \\
& & \\
BMI $\left(\mathrm{kg} / \mathrm{m}^{2}\right)$ & $23.18 \pm 4.92$ & $22.19 \pm 1.68$
\end{tabular}

All data are expressed as means with standard deviation (SD).

\subsection{Method to Induce DOMS}

DOMS was inducted from the non-dominant arm, the left arm, so that the subjects' ordinary life was not impeded.

One repetition maximum (1RM) method, the maximum amount of weight one can lift in a single repetition for a given exercise, was used in order to induce DOMS[3]. The subject in front of the treatment table flexed the shoulder joint at 45 degrees, placed the elbow on the pad of the table, grabbed a dumbbell weighing $60 \%$ of the $1 \mathrm{RM}$, maintained it for one second at 90 degrees, and lowered it slowly for three seconds. After the elbow was completely extended, the experiment or manually made the subject's arm in a 90 degree flexion position (Figure1).

Using such method, the subject conducted eccentric exercise for 5 sets with 15 times as one set. When the subject did not maintain extension of the elbow for eight seconds, the exercise was finished. The subject took a rest for one minute each time [22].

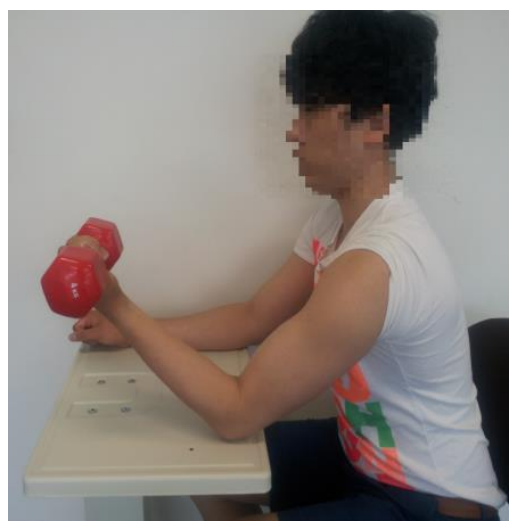

Figure1. Eccentric Exercise for Biceps Brachii to Induce DOMS 


\subsection{Application of Taping}

As an elastic tape for pain control of group II, Kinesio tape (Kinesio TEX; Kinesio TAPING Co. Ltd. JAPAN) was used. In order to minimize errors, the same person attached the taping on the subjects.

The subjects sitting on a chair placed the upper arm on the treatment table and the tape was applied along the biceps brachii of the non-dominant side from DOMS was induced. The tape was tightly attached to the skin area where it was applied(Figure 2.).

It was maximally lengthened. Stimulation was made once per day, which was the recommended amount in the clinical field. An inelastic tape with a similar color to that of Kinesio tape was attached to the group I.

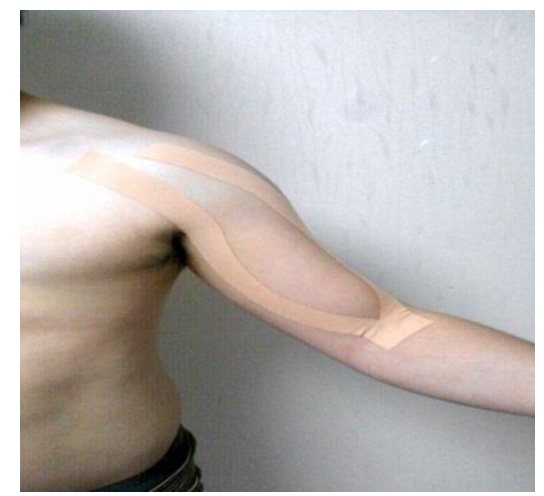

\section{Figure 2. Application of Kinesio Taping on Biceps Brachii}

\subsection{Evaluation Tool and Methods}

TSA-2001 thermal sensory analyzer (TSA-II, MEDOC Inc, Israel) (Figure 3.)was used in order to examine changes in pain through QST. It was conducted in a comfortable space with no obstruction.

The subjects in a sitting position fully extended the elbow joint on the measurement table and the central area of the biceps brachii muscle belly was measured with an thermode. The reference temperature was set and temperature was increased or decreased by $1^{\circ} \mathrm{C}$ per second and CST and WST were measured. CPT and WPT, which were to measure pain felt when stimulation was given, were measured with the subjects pressing the button themselves.

The reference temperature, maximal temperature, and minimal temperature were $30{ }^{\circ} \mathrm{C}, 50$ ${ }^{\circ} \mathrm{C}$, and $0{ }^{\circ} \mathrm{C}$. When the subjects did not feel cold sensation and cold pain, the temperature was measured to be $0{ }^{\circ} \mathrm{C}$ and when they did not feel warm sensation and warm pain, the temperature was measured to be $50{ }^{\circ} \mathrm{C}$.

Sensation thresholds were measured four times with a resting time ranging from four to six seconds and pain threshold were measured three times with a resting time for 10 seconds [23]. Matters that require attention were sufficiently explained to the subjects and the intervention was made after some practice.

Visual Analog Scale (VAS) was used to look at changes in subjective pain. A straight line of $10 \mathrm{~cm}$ was drawn with one end marked as no pain and the other end marked as unbearable pain. For more segmented values, the straight line was divided into sections. Each section was determined to be $1 \mathrm{~mm}$.

The subjects marked themselves their subject pain after listening to sufficient explanation [24]. All measurement was taken prior to the intervention, 24 hours after the intervention, 48 hours after the intervention, and 72 hours after the intervention. 


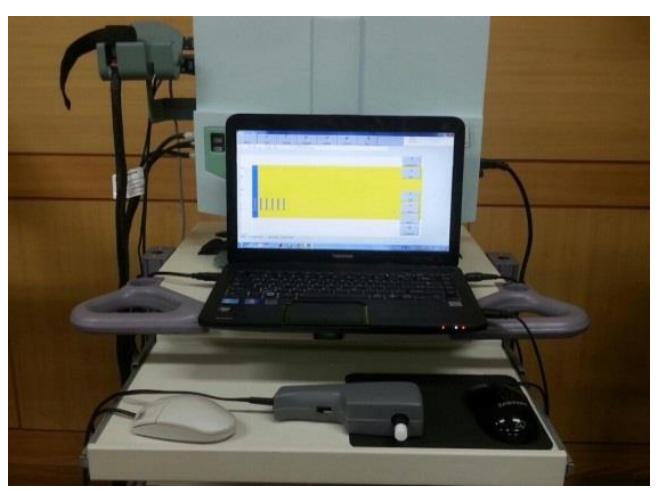

Figure 3. TSA-2001 Thermal Sensory Analyzer (TSA-II, MEDOC Inc, Israel)

\subsection{Statistical Analysis}

The mean and standard deviation were calculated using SPSS 18.0 Window version. Repeated measure analysis of variance (ANOVA) was conducted in order to analyze changes within each group according to four measured time points. Scheffe post hoc test was performed and an independent $t$-test was carried out in order to analyze differences between the two groups. A statistical significance level was set at $\alpha=0.05$.

\section{Results}

After artificial inducement of DOMS, Kinesio-taping was applied and thermal pain threshold (WPT, CPT) was measured at each measurement time point. There were significant differences according to the measurement time point in all the groups $(p<0.001)$. Pain decreased from 24 hours after inducement of DOMS in group II (Table 2).

According to QST made at each measured time point after DOMS induction and Kinesio taping application, the two groups' CPT, WPT, and CST decreased 24 hours after DOMS induction compared to prior to DOMS induction $(\mathrm{p}<0.05)$. Their decreases were greatest 48 hours after DOMS induction ( $\mathrm{p}<0.05)$. From 48 hours after DOMS induction, the two groups' CPT, WPT, and CST increased over time.

\section{Table 2. The Changes of Thermal Pain Threshold (TPT) through Quantitative Sensory Testing (QST) $\left({ }^{\circ} \mathrm{C}\right)$}

\begin{tabular}{cccccccc}
\hline & & $-24 \mathrm{~h}(\mathrm{a})$ & $24 \mathrm{~h}(\mathrm{~b})$ & $48 \mathrm{~h}(\mathrm{c})$ & $72 \mathrm{~h}(\mathrm{~d})$ & $\mathrm{P}$ & $\begin{array}{c}\text { post-hoc } \\
\text { contrasts }\end{array}$ \\
\hline group I & CPT & $16.15 \pm 2.25$ & $7.49 \pm 2.94$ & $5.95 \pm 2.83$ & $8.86 \pm 2.21$ & $<.001$ & $\mathrm{ab}, \mathrm{ac}, \mathrm{ad}$ \\
& WPT & $38.63 \pm 3.14$ & $29.62 \pm 3.28$ & $28.56 \pm 3.35$ & $30.19 \pm 3.57$ & $<.001$ & $\mathrm{ab}, \mathrm{ac}, \mathrm{ad}$ \\
\multirow{3}{*}{ group II } & CPT & $15.79 \pm 1.61$ & $10.57 \pm 2.44$ & $7.07 \pm 3.21$ & $14.35 \pm 2.60^{\#}$ & $<.001$ & $\mathrm{ab}, \mathrm{ac}$, \\
& WPT & $39.17 \pm 2.81$ & $36.67 \pm 3.85$ & $33.83 \pm 4.21$ & $38.99 \pm 3.76^{\#}$ & $<.001$ & $\mathrm{ab}, \mathrm{ac}$ \\
& & & & & & & \\
\hline
\end{tabular}

All Values showed mean \pm S.D

-24h: 24 hours prior to DOMS, 24h: 24 hours after DOMS, 48h: 48 hours after DOMS, $72 \mathrm{~h}: 72$ hours after DOMS, CPT: Cold pain threshold, WPT: Warm pain threshold, ab:-24h*24h, ac:$24{ }^{*} 48 h$, ad: $-24 h * 72 h$ by repeated measure ANOVA *: Independent $t$-test $(p<0.05)$ 


\section{Table 3. The Changes of Thermal Sensation Threshold Through Quantitative} Sensory Testing (QST) $\left({ }^{\circ} \mathrm{C}\right)$

\begin{tabular}{|c|c|c|c|c|c|c|c|}
\hline & & $-24 h(a)$ & $24 h(b)$ & $48 \mathrm{~h}(\mathrm{c})$ & $72 \mathrm{~h}(\mathrm{~d})$ & $\mathrm{P}$ & $\begin{array}{l}\text { post-hoc } \\
\text { contrasts }\end{array}$ \\
\hline \multirow[t]{2}{*}{ group I } & CST & $29.39 \pm 0.95$ & $24.68 \pm 2.21$ & $18.68 \pm 2.55$ & $23.50 \pm 2.60$ & $<.001$ & $a b, a c, a d$ \\
\hline & WST & $34.73 \pm 1.34$ & $42.26 \pm 1.24$ & $45.44 \pm 4.15$ & $39.61 \pm 2.65$ & $<.001$ & $\mathrm{ab}, \mathrm{ac}, \mathrm{ad}$ \\
\hline \multirow[t]{2}{*}{ group II } & CST & $29.39 \pm 1.85$ & $26.68 \pm 3.36$ & $22.83 \pm 3.32$ & $28.90 \pm 1.94$ & $<.001$ & $a b, a c$, \\
\hline & WST & $33.14 \pm 1.87$ & $37.98 \pm 2.09$ & $38.27 \pm 2.74$ & $35.30 \pm 1.99$ & $<.001$ & $\mathrm{ab}, \mathrm{ac}$ \\
\hline
\end{tabular}

All Values showed mean \pm S.D.

-24h: 24 hours prior to DOMS, 24h: 24 hours after DOMS, 48h: 48 hours after DOMS, $72 \mathrm{~h}: 72$ hours after DOMS, CST: Cold Sensation Threshold, WST: Warm Sensation threshold, ab:$24 h^{*} 24 h$, ac: $-24 h^{*} 48 h$, ad: $-24 h^{*} 72 h$ by repeated measure ANOVA \#: Independent $t-$ test $(\mathrm{p}<0.05)$

Among them, the values of the group II to which Kinesio taping was applied were greater. The values prior to DOMS induction were not significantly different from those 72 hours after DOMS induction ( $\mathrm{p}>0.05)$. The two groups' WST went up 24 hours after DOMS induction compared to prior to DOMS induction $(\mathrm{p}<0.05)$ and was greatest 48 hours after DOMS induction $(\mathrm{p}<0.05)$.

It decreased from 48 hours after DOMS induction over time; The WST of the group II 72 hours after DOMS induction was not statistically significantly different from that prior to DOMS induction ( $>0.05)$. When the two groups were compared, there were statistically significant differences in the CST and WST 72 hours after DOMS induction (Table 3).

VAS according to measured time point was measured and it started from 24 hours after DOMS induction and became greatest 48 hours after DOMS induction. In comparison between 24 hours after DOMS induction and 72 hours after DOMS induction, there were statistically significant decreases in pain $(\mathrm{p}<0.05)($ Table 4$)$.

Table 4. The Changes of Muscle VAS Score

\begin{tabular}{ccccccc}
\hline & $-24 \mathrm{~h}(\mathrm{a})$ & $24 \mathrm{~h}(\mathrm{~b})$ & $48(\mathrm{c})$ & $72 \mathrm{~h}(\mathrm{~d})$ & $\mathrm{P}$ & post-hoc contrasts \\
\hline group I & 0 & $5.30 \pm 1.41$ & $5.70 \pm 0.76$ & $2.60 \pm 1.47$ & $<0.01$ & bd \\
& & & & & & bd \\
group II & 0 & $4.90 \pm 1.59$ & $5.60 \pm 0.46$ & $2.10 \pm 0.98$ & $<0.01$ & \\
\hline
\end{tabular}

All Values showed mean \pm S.D.

-24h: 24 hours prior to DOMS, 24h: 24 hours after DOMS, $48 \mathrm{~h}: 48$ hours after DOMS, $72 \mathrm{~h}: 72$

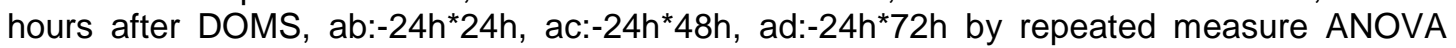
$(p<0.05)$

\section{Discussion}

Many people experience pain due to diverse causes in their ordinary life. In particular, sudden exercise and repetition of unfamiliar motions trigger DOMS. DOMS is apparent in the 
muscle belly and the musculotendinous junction and it accompanies micromechanical damage of the muscles.

As inflammatory responses after primary micromechanical damage of the muscles, chemical materials such as histamine, bradykinin, and prostaglandin are released, and increased intramuscular penetration raises osmotic pressure. As a result, afferent nerve fibers are activated and pain is delivered to the central nervous system [25]. It also disturbs adenosine triphosphate reproduction within the muscles and decreases calcium ion release in the contraction mechanism, lowering contraction function.

In particular, DOMS occurs more frequently during strong eccentric motions than concentric motions; Eccentric contraction is largely used in research concerning induction of DOMS because muscle fiber rupture often takes places from such eccentric contraction [26].

Kinesio taping has been commonly used for protection and strengthening of the joints by application to the skins or muscles and for pain decrease, prevention of additional damage, and reduction in edema when the musculoskeletal system was acutely damaged [27]. Further, Kinesio taping is used as a main or assistant treatment method in diverse clinical fields, for example, in order to treat osteoarthritis or knee joint pain, helping patients conduct their ordinary life[19, 28].

Objective and specific evaluation is very important for the treatment of patients. However, pain is a complex and subjective phenomenon and objective approach in its evaluation is also difficult. Due to intra-rater and inter-rater differences, the limitation of subjective evaluation, such evaluation should be objectified through QST on temperature sensation and pain.

QST is a useful method to study pain and functional abnormality induced by stimuli and physiological characteristics of sensory receptors[29]. Relative to ordinary clinical scales, QST has high sensitivity and reproductivity and is very useful[30]. It also may be of use in obtaining efficiency, validity, and reliability of treatment. QST is a method to determine absolute sensory threshold for precise perception of special kinds of stimuli; It enables objective examination on patients with abnormality in the sensory nerve system through quantification and may investigate whether there is abnormality in the concentric paths of somatic senses[31].

Accordingly, this study intended to objectively examine the effects of Kinesio taping according to measured time point on DOMS, which had been evaluated subjectively, through QST. Cutaneous fusimotor reflex induces muscle contraction, adjusts the degree of muscle tone, and maintains voluntary balance between muscles, making the body in an ideal condition.

When muscles contract, lymphatic and blood circulation increases. Kinesio taping makes use of cutaneous fusimotor reflex, a phenomenon where muscles under the skin to which it is attached continuously contract. Pain receptors are connected to A $\delta$ fibers, myelinated nerves with a small diameter, and psychological and physiological characteristics of patients with pain stimulate the sympathetic nervous system, reducing blood flow on the body surface and lowering body temperature [32]. There has been research that reported the more intense pain was, the lower pain threshold for thermal stimuli [33].

This study looked at the effects of Kinesio taping after the induction of DOMS on objective sensory changes through QST. Sensation thresholds(WPT, CPT, WST, CST) of all the groups became significantly different from 24 hours after the intervention. The two groups WST went up 24 hours after DOMS induction compared to prior to DOMS induction $(p<0.05)$ and was greatest 48 hours after DOMS induction $(p<0.05)$. Significant differences $(p<0.05)$ in all the groups 48 hours after the intervention showed that pain increased.

In the group II to which Kinesio taping was applied, there was no significant difference between 72 hours after DOMS induction and prior to DOMS induction ( $p>0.05)$, showing the 
effects of Kinesio taping(Table 3). In comparison between the two groups, CPT and WPT were significantly different between the two groups 72 hours after the intervention $(\mathrm{p}>0.05)$ (Table 2). CPT and WPT, which represent pain thresholds, more increased in the group II than the group I, signifying that Kinesio taping was a useful intervention to reduce pain after the induction of DOMS.

This is considered because Kinesio-taping attached along the muscles pulled them and stimulated the golgi tendon organ, and generated autogenic inhibition signals. In addition, it is regarded that promotion of metabolism caused by the induction of muscle contraction decreased pain. It is considered that stimulation of vasomotor reflex increases blood circulation and helps secretion of various matters that may heighten muscle temperature.

In order to examine changes in sensation threshold after the treatment, this study calculated difference between CPT and HPT, temperatures one started to perceive as cold and warm stimuli; This value is a range when the patients do not perceive warm and cold stimuli as pain and their sensitivity to pain was able to be evaluated.

VAS is usually used to assess overall intensity of pain but may also be employed to evaluate the intensity or frequency of pain[24]. VAS is easy and fast to use and score, and can minimize an experimenter's involvement. However, it cannot take into account diverse aspect of pain at the same time. Sharp stinging pain and heavy and stiff pain are obviously different but VAS does not differentiate them.

VAS was measured in order to look at the effects of Kinesio taping on subjective pain index; It started to go up 24 hours after DOMS induction and became greatest 48 hours after DOMS induction. In the group II, there was statistically significant pain decrease in comparison between 24 hours after DOMS induction and 72 hours after DOMS induction $(\mathrm{p}<0.05)($ Table 4). This shows that taping influences subjective pain decrease. This is considered a mechanism that may occur due to psychological influence as well as the effect of elastic Kinesio taping [34].

Efforts to relieve pain are an essential task in the clinical field and in order to effectively achieve this task, side effects from pain should be minimized in applying treatment to patients [35]. Therefore, diagnosis and evaluation of pain is a clinically very crucial task. Pain is affected by various subjective factors and therefore diagnosis and evaluation of pain is very difficult.

QST is much used in order to define the degree of pain or control pain. As stimuli for QST, heat, cold, electrical, and mechanical stimuli have been used[36]. Among others, temperature threshold test is an indispensible test item in QST protocol and accepted as a reliable test[21].

Nonetheless, most existing research assessed pain with a subjective tool. On the contrary, this study utilized QST to quantify pain and identify the aspects of changes in pain. Such objective data is considered to be very meaningful as clinical basic data. It will be greatly helpful in objectively evaluating pain of patients and predicting and treating the degree of actual pain they may feel.

The limitation of this study is a small number of samples: It also did not research women, although it had been reported that there was no difference in DOMS between genders [37]. Accordingly, research on a diversity of subjects will be necessary. Further, this study concerned adults in their 20s and research on a wide range of age groups is needed for objectifying the result. Besides, continuous observation after 72 hours after DOMS induction is necessary. 


\section{References}

[1] K. Cheung, P. A. Hume and L. Maxwell, J. Sports Med., vol. 33, (2003), pp. 145.

[2] M. PMiles and P. M. Clarkson, J. Sports Med Phys Fitness, vol. 34, (1994), pp. 203.

[3] C . Kisner and L. A. Colby, "Therapeutic Exercise: Foundations and techniques", Edited PA, F. A. Davis Co, Philadelphia, vol. 5, (2007), pp147-229.

[4] K. L. Sellwood, P. Brukner, D. Williams, A. Nicol and R. Hinman, Br J. Sports Med., vol. 41, (2007), p. 392.

[5] K. P. O'Reilly, M. J. Warhol, R. A. Fielding, W. R. Frontera, C. N. Meredith and W. J. Evans, J. Appl Physiol., vol. 63, (1987), p. 252.

[6] J. Friden, M. Sjostrom and B. Ekblom, J. Sports Med., vol. 4, (1983), p. 170.

[7] R. L. Lieber and J. Friden, J. Am Acad Orthop Surg., vol. 10, (2002), p. 67.

[8] K. Nosaka and M. Newton, J. Med Sci Sports Exerc., vol. 34, (2002), p. 63.

[9] D. A. Connolly, S. P. Sayers, M. P. McHugh, J. Strength Cond Res., vol. 17, (2003), p. 197.

[10] R. Torres, F. Ribeiro, Alberto Duarte J and J. M. Cabri, J._Phys Ther Sport, vol. 13, (2012), p. 101.

[11] G. L. Warren, D. A. Lowe and R. B. Armstrong, J. Sports Med., vol. 27, (1999), p. 43.

[12] C. M. Alexander, M. McMullan, and P. J. Harrison, Manual Therapy, vol. 13, (2008), p. 57.

[13] F. Garcia-Muro, A. L. Rodriguez-Fernandez, and A. Herrero-de-Lucas, Manual Therapy, vol. 15, (2010), p. 292.

[14] T. Halseth, J. W. McChesney, M. DeBeliso, R. Vaughn and J. Lien, J. Sports Sci Med., vol. 3, (2004), p. 1.

[15] H. H. Host, Physical Therapy, vol. 75, (1995), p. 803.

[16] Y. R. Choi, B. J. Jung and B. J. Hwang, J. Korean Soc Radiol, vol. 7, (2013), p. 99.

[17] C. D. Black and R. M. Dobson, Clin J Pain, vol. 29, (2013), p. 443.

[18] J. Vaile, S. Halson, N. Gill and B. Dawson, Eur J. Appl Physiol, vol. 102, (2008), p. 447.

[19] G. L. Wasner and J. A. Brock, Clin Neurophysiol, vol. 119, (2009), p. 2389.

[20] M. Pigg, L. Baad-Hansen, P. Svensson, M. Drangsholt and T. List, Pain, vol. 148, (2010), p. 220.

[21] M. Pigg, P. Svensson and T. List, J. Orofacl Pain, vol. 25, (2011), p. 39.

[22] D. G. Behm, K. M. Baker, R. Kelland, and J. Lomond, J. Strength Cond Res., vol. 15, (2001), p. 255.

[23] J. B. Shin, K. J. Cho, S. G. Jang, S. You, Y. J. Seong and J. R. Park, J. of Korean Acad. of Rehab. Med., vol. 24, (2000), p. 1225.

[24] A. M. Boonstra, H. R. Schiphorst Preuper, M. F. Reneman, J. B. Posthumus and R. E. Stewart, Int J Rehabil Res., vol. 31, (2008), p. 165.

[25] P. M. Clarkson and M. J. Hubal, Am J Phys Med Rehabil, vol. 82, (2002), p. 52.

[26] K. Nasaka, K. Sakamoto, M, Newton and P. Sacco, EurJ Appl Physiol, vol. 85, (2001), p. 34.

[27] J. Leanderson, S. Ekstam, and C. Salomonsson, Knee Surq Sports Traumatol Arthrosc, vol. 4, (1996), p. 53.

[28] G. Bálint and B. Szebenyi, Baillieres Clin Rheumatol, vol. 11, (1997), p. 795.

[29] G. L. Shelton, Primary Care, vol. 19, (1992), p. 331.

[30] V. S. Erickson, M. L. Pearson, P. A. Ganz, J. Adams, and K. L. Kahn, J Natl Cancer Inst., vol. 93, (2001), p. 96.

[31] S. H. Bae, Y. S. Lee, G. D. Kim and K. Y. Kim, Advanced Science and Technology Letters (ASTL), vol. 47, (2014), p. 387.

[32] E. E. Benarroch, Clin Auton Res., vol. 11, (2001), p. 343.

[33] W. M. Hooten, P. Sandroni, C. B. Mantilla and C. O. Townsend, Pain Med., vol. 11, (2010), p. 1554

[34] M. Smith, V. Sparkes, M. Busse, and S. Enright, Phys Ther Sport, vol. 10, (2009), p. 45.

[35] A. Caraceni, N. Cherny, R. Fainsinger, S. Kaasa, P. Poulain, L. Radbruch and F. De Conno, J Pain Symptom Manage, vol. 23, (2002), p. 239.

[36] T. J. Neddermeyer, K. Flühr and J. Lötsch, Pain, vol. 138, (2008), p. 286.

[37] M. J. Hubal, and P. M.Clarkson, J Appl Physiol, vol. 106, (2009), p. 101. 


\section{Authors}

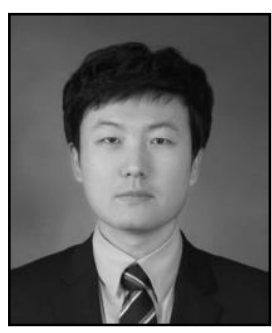

Sea-Hyun Bae

February 2013: Dept. of Physical Therapy, Dongshin University(M.S.)

February 2014: Dept. of Physical Therapy, Dongshin University(Ph.D.)

March 2013 Current: Dept. of Physical Therapy, Cheongam

College(Visiting Professor)

$<$ Interests >: Neurological physical therapy, Clinical electrophysiological

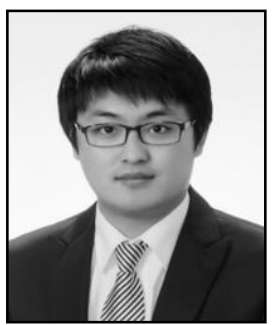

Young-Shin Lee

February 2013: Dept. of Physical Therapy, Dongshin University(M.S.) March 2013 Current: Dept. of Physical Therapy, Dongshin University(Ph.D. course)

<Interests>: Neuroscience, Neurological physical therapy

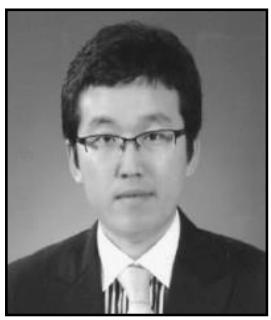

Gi-Do Kim

February 2004: Dept. of Physical Therapy, Dongshin University(M.S.) February 2010: Dept. of Physical Therapy, Dongshin University(Ph.D.) March 2011 Current: Dept. of Physical Therapy, International University(Professor)

<Interests>: Neuroscience, Clinical electrophysiological

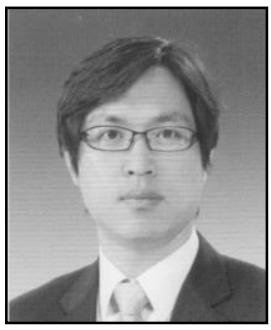

\section{Kyun-Yoon Kim}

February 2004: Dept. of Physical Therapy, Dongshin University(M.S.)

February 2007: Dept. of Physical Therapy, Dongshin University(Ph.D.) March 2006 Current: Dept. of Physical Therapy, Dongshin University(Professor)

$<$ Interests $>$ : Neuroscience, Neurological physical therapy 